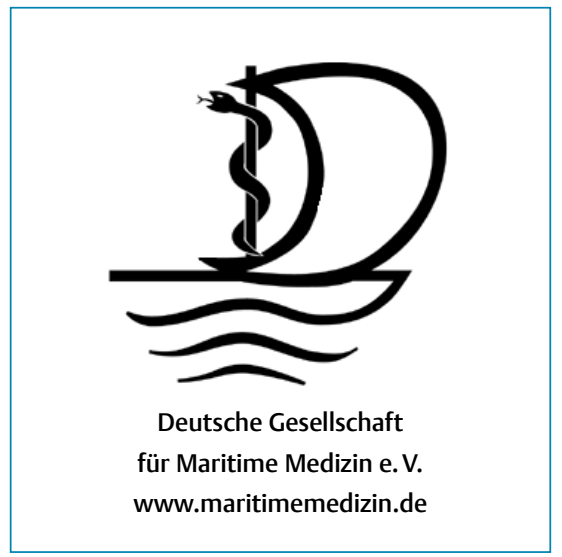

Museumsschiff Cap San Diego, Hamburg.

(c) Cap San Diego

\title{
Mitgliederversammlung 2011
}

Am 20. Januar 2012 fand die Mitgliederversammlung 2011 der Deutschen Gesellschaft für Maritime Medizin (DGMM) statt - traditionsgemäß wieder im Salon der Cap San Diego, Hamburg, Überseebrücke. Ein detailliertes Protokoll der Versammlung haben die Mitglieder der DGMM satzungsgemäß bereits erhalten. Nachfolgend werden einige Punkte der Tagesordnung für den erweiterten Leserkreis nochmals zusammengefasst:

Nachdem die vom Vorsitzenden herzlich begrüßten 45 erschienenen Mitglieder die Tagesordnung sowie das Protokoll der Mitgliederversammlung vom 28. Januar 2011 einstimmig gebilligt hatten, konnte die Mitgliederversammlung 42 neue Mitglieder aufnehmen. Damit hat die DGMM nunmehr 201 ordentliche Mitglieder.

\section{Vorstandsarbeit 2011}

Der Vorsitzende berichtete über die Inhalte der Vorstandsarbeit und die durchgeführten Vorstandssitzungen am 27. April und 7. Dezember 2011.

Das Amtsgericht Hamburg (Registergericht) stimmte inzwischen allen Satzungsänderungen, die von der Mitgliederversammlung beschlossen worden waren, zu und bestätigte damit auch die auf der letzten Mitgliederversammlung in getrennten Wahlgängen durchgeführte Neuwahl aller Vorstandsmitglieder.

Die DGMM-Homepage hat sich unter der Betreuung durch Dr. Klaus-H. Seidenstücker deutlich weiterentwickelt und verbessert, diverse zusätzliche Links wurden eingefügt, unter anderem sind alle DGMM-Mitteilungen aus der FTR des letzten Kalenderjahrs dort aufrufbar. Be- sonders wies der Vorsitzende auf den Link und die Internetadresse zur Zeitschrift „International Maritime Health“, www.intmarhealth.pl, hin. Sämtliche Artikel dieses Journals der International Maritime Health Association/IMHA können kostenlos aufgerufen werden. In diesem Zusammenhang warb der Vorsitzende erneut für eine Mitgliedschaft auch in der IMHA, da maritime Medizin immer mehr - wie die Seeschifffahrt selbst - international wird.

Das DGMM-Mitgliederverzeichnis, das aus Kostengründen in Papierform beschlossen wurde, ist bei Christoph Sevenich in Bearbeitung. Der Rücklauf der an die Mitglieder versandten Fragebögen lag Anfang Dezember 2011 bei circa 65\%. Sofern kein Fragebogen ausgefüllt zurückgesandt wurde, wird im Mitgliederverzeichnis nur der Name des Mitglieds ohne ergänzende Information aufgeführt sein. In das Verzeichnis sollen die $42 \mathrm{Neu}-$ aufnahmen dieser Mitgliederversammlung mit aufgenommen werden.

Die seit Dezember 2010 fertig gestellte und veröffentlichte DGMM-Empfehlung „Ärztliche Eignungsuntersuchungen bei Arbeitnehmern auf Offshore-Windenergieanlagen und Plattformen" hat eine gute Resonanz gefunden. Betriebsärzte führen die entsprechenden Untersuchungen gemäß dieser Empfehlung durch. Auch eine englische Übersetzung wurde inzwischen gefertigt. Die DGMM-Arbeitsgruppe Offshore-Medizin, Sprecherin Dr. Alexandra Preisser, wird im Jahr 2012 prüfen, ob beziehungsweise wie eine Überarbeitung der Empfehlung angezeigt erscheint. Zusätzlich befasst sich diese 
Arbeitsgruppe bereits mit der Entwicklung eines Konzepts zur medizinischen Ausbildung des Offshorepersonals sowie zur medizinischen Ausstattung von Offshore-Anlagen beziehungsweise -plattformen. Da es ein derartiges allgemeingültigeres Konzept bisher in Deutschland nicht gibt, machen sich derzeit hierzu etliche Firmen eigene Gedanken, ohne sich untereinander abzustimmen.

Da sich die DGMM der offshoremedizinischen Fortbildung der Arbeitsmediziner verpflichtet fühlt, fand am 2./3. September 2011 der 2. Emder Workshop mit dieser Zielrichtung statt, organisiert von VGB Power Tech in Zusammenarbeit mit der DGMM. VGB Power Tech führt derzeit eine Liste der Ärzte, die bereits Erfahrungen mit Offshore-Eignungsuntersuchungen gesammelt und sich entsprechend fortgebildet haben. Ein Bericht über den 2. Emder Workshop war in der FTR 6/2011 zu lesen. Über den DGMM-Einführungslehrgang „Maritime Notfallmedizin“, durchgeführt am 18./19. November 2011 im HPHC in Hamburg auf der Grundlage des entsprechenden DGMM-Curriculums, wurde in der FTR 1/2012 berichtet. Ein weiterer Lehrgang ist für Mitte November 2012 geplant.

Die veränderte Klientel des Einführungslehrgangs, der voraussichtliche Wegfall des Zertifikats „Maritime Medizin“ der Ärztekammer Schleswig-Holstein, wie auch Ausbildungsinitiativen verschiedener Anbieter gaben dem Vorstand Anlass zur Einsetzung einer Arbeitsgruppe. Diese soll Kriterien erarbeiten, auf deren Grundlage die DGMM zu einer Empfehlung für eine angemessene Qualifikation von Schiffsärzten und gegebenenfalls auch für die Zertifizierung entsprechender Weiter- und Fortbildungsangebote befähigt wird.

Das Projekt „Geschichte der Schifffahrtsmedizin in Deutschland nach 1945“ ist in 3 Anteilen geplant: einem ostdeutschen Teil der Zeit 1945-1990, einem westdeutschen Teil der gleichen Zeitspanne und einem gesamtdeutschen Teil ab 1990. Prof. Dr. Heinz Ebert hat bereits den ostdeutschen Teil fertig gestellt. Dieser soll allen Mitgliedern in gedruckter Form zur Verfügung gestellt werden.

Zum „Pocket Guide for Cold Water Survival“ der IMO hatte Vorstandsmitglied Dr. Jens Kohfahl eine aktuelle Literaturrecherche durchgeführt und einige Änderungsvorschläge formuliert, die als Vorschlag der DGMM über das BMVBS der

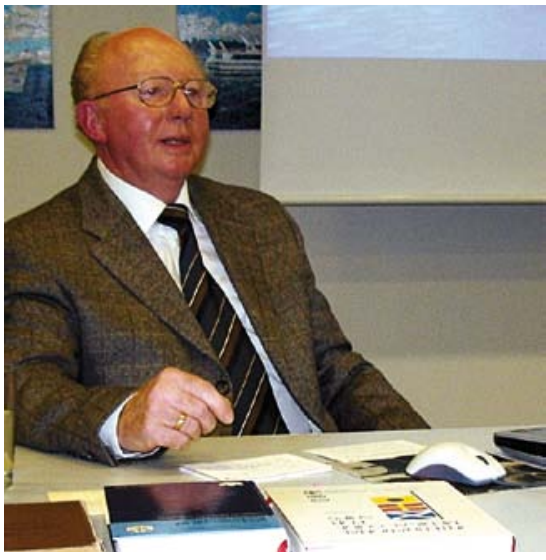

Ehrenmitglied Prof. Dr. Heinz Ebert.

IMO zugeleitet wurden. Die Vorschläge fanden auf der hierfür zuständigen COMSAR-Sitzung der IMO im März 2011 ganz überwiegende Zustimmung und sollen auf der COMSAR-Sitzung im März 2012, an der Dr. Jens Kohfahl als Repräsentant der DGMM und gleichzeitig Berater des BMVBS erneut persönlich teilnehmen wird, beschlossen werden.

Zum Abschluss seines Berichts bedankte sich der Vorsitzende bei seinen Vorstandskollegen für ihren Einsatz und die kollegiale Zusammenarbeit im abgelaufenen Jahr.

\section{Entlastung}

Nach dem Bericht des Schatzmeisters für das Geschäftsjahr 2011 und dem Bericht der Kassenprüfer wurde der Vorstand einstimmig entlastet.

\section{Beschlüsse}

Die Mitgliederversammlung beschloss einstimmig 2 vom Vorstand vorbereitete und mit der Einladung zugeleitete Satzungsänderungen:

- Die Aufnahme neuer Mitglieder wird zukünftig Aufgabe des Vorstands sein.

- Die Beschlussfähigkeit der Mitgliederversammlung wird auch bei stark gestiegener Mitgliederzahl gewährleistet.

Abgerundet wurde die Mitgliederversammlung durch einen Fortbildungsvortrag des DGMM-Ehrenmitglieds Prof. Dr. Heinz Ebert zum Thema „Maritime Medizin in der ehemaligen DDR 1945-1990 ein Stück deutscher Geschichte“, der ein reges Interesse fand.

Abschließend bestand wie immer die Möglichkeit zu einem gemeinsamen Essen im Speisesalon der Cap San Diego samt geselligem Beisammensein an Bord.

Dr. Bernd-Fred Schepers,

Vorsitzender der DGMM, Hamburg 